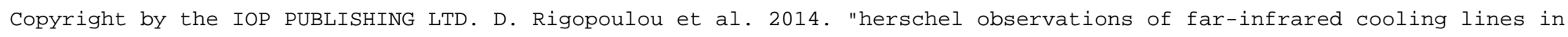
intermediate redshift (ultra)-luminous infrared galaxies," ApJ 781 L15 doi:10.1088/2041-8205/781/1/L15

The Astrophysical Journal LetTers, 781:L15 (5pp), 2014 January 20

doi:10.1088/2041-8205/781/1/L15

(C) 2014. The American Astronomical Society. All rights reserved. Printed in the U.S.A.

\title{
HERSCHEL OBSERVATIONS OF FAR-INFRARED COOLING LINES IN INTERMEDIATE REDSHIFT (ULTRA)-LUMINOUS INFRARED GALAXIES*
}

\author{
D. Rigopoulou ${ }^{1,2}$, R. Hopwood ${ }^{3}$, G. E. Magdis ${ }^{1}$, N. Thatte ${ }^{1}$, B. M. Swinyard ${ }^{2,4}$, D. FArRAh ${ }^{5}$, J.-S. HuAng ${ }^{6,7,8}$, \\ A. Alonso-Herrero 9 , J. J. Bock ${ }^{10,11}$, D. Clements ${ }^{3}$, A. Cooray ${ }^{11,12}$, M. J. Griffin ${ }^{13}$, S. Oliver ${ }^{14}$, C. Pearson $^{2,15}$, \\ D. Riechers ${ }^{16}$, D. ScotT ${ }^{17}$, A. Smith ${ }^{14}$, M. Vaccari ${ }^{18}$, I. Valtchanov $^{19}$, And L. Wang ${ }^{14}$ \\ ${ }^{1}$ Department of Physics, University of Oxford, Keble Road, Oxford OX1 3RH, UK \\ ${ }^{2}$ RAL Space, Science, and Technology Facilities Council, Rutherford Appleton Laboratory, Didcot OX11 0QX, UK \\ ${ }^{3}$ Physics Department, Imperial College London, South Kensington Campus, London SW7 2AZ, UK \\ ${ }^{4}$ Department of Physics and Astronomy, University College London, Gower Street, London WC1E 6BT, UK \\ 5 Department of Physics, Virginia Tech, Blacksburg, VA 24061, USA \\ ${ }^{6}$ National Astronomical Observatories of China, Chinese Academy of Sciences, Beijing 100012, China \\ ${ }^{7}$ China-Chile Joint Center for Astronomy, Chinese Academy of Sciences, Camino El Observatorio, \#1515, Las Condes, Santiago, Chile \\ ${ }^{8}$ Harvard-Smithsonian Center for Astrophysics, 60 Garden Street, Cambridge, MA 02138, USA \\ ${ }^{9}$ Instituto de Fisica de Cantabria, CSIC-UC, E-39006 Santander, Spain \\ ${ }^{10}$ California Institute of Technology, 1200 E. California Blvd., Pasadena, CA 91125, USA \\ ${ }^{11}$ Jet Propulsion Laboratory, 4800 Oak Grove Drive, Pasadena, CA 91109, USA \\ ${ }^{12}$ Department of Physics and Astronomy, University of California, Irvine, CA 92697, USA \\ ${ }^{13}$ School of Physics and Astronomy, Cardiff University, Queens Buildings, The Parade, Cardiff CF24 3AA, UK \\ ${ }^{14}$ Astronomy Centre, Department of Physics and Astronomy, University of Sussex, Brighton BN1 9QH, UK \\ ${ }^{15}$ Department of Physical Sciences, The Open University, Milton Keynes MK7 6AA, UK \\ ${ }^{16}$ Department of Astronomy, Cornell University, 220 Space Sciences Building, Ithaca, NY 14853, USA \\ ${ }^{17}$ Department of Physics and Astronomy, University of British Columbia, 6224 Agricultural Road, Vancouver, BC V6T1Z1, Canada \\ ${ }^{18}$ Astrophysics Group, Physics Department, University of the Western Cape, Private Bag X17, 7535 Bellville, Cape Town, South Africa \\ ${ }_{19}^{19}$ Herschel Science Centre, European Space Astronomy Centre, Villanueva de la Canada, E-28691 Madrid, Spain \\ Received 2013 October 11; accepted 2013 December 6; published 2014 January 6
}

\begin{abstract}
We report the first results from a spectroscopic survey of the [C II $] 158 \mu \mathrm{m}$ line from a sample of intermediate redshift $(0.2<z<0.8)$ (ultra)-luminous infrared galaxies, (U)LIRGs $\left(L_{\mathrm{IR}}>10^{11.5} L_{\odot}\right)$, using the Spectral and Photometric Imaging REceiver-Fourier Transform Spectrometer on board the Herschel Space Observatory. This is the first survey of [C II] emission, an important tracer of star formation, at a redshift range where the star formation rate density of the universe increases rapidly. We detect strong [C II] $158 \mu \mathrm{m}$ line emission from over $80 \%$ of the sample. We find that the $\left[\mathrm{C}_{\mathrm{II}}\right]$ line is luminous, in the range $(0.8-4) \times 10^{-3}$ of the far-infrared continuum luminosity of our sources, and appears to arise from photodissociation regions on the surface of molecular clouds. The $L_{[\mathrm{C}}{ }_{\text {II }} / L_{\mathrm{IR}}$ ratio in our intermediate redshift (U)LIRGs is on average $\sim 10$ times larger than that of local ULIRGs. Furthermore, we find that the $L_{[\mathrm{C} \text { II] }} / L_{\mathrm{IR}}$ and $L_{[\mathrm{C} \text { II] }} / L_{\mathrm{CO}(1-0)}$ ratios in our sample are similar to those of local normal galaxies and high- $z$ star-forming galaxies. ULIRGs at $z \sim 0.5$ show many similarities to the properties of local normal and high- $z$ star-forming galaxies. Our findings strongly suggest that rapid evolution in the properties of the star-forming regions of (U)LIRGs is likely to have occurred in the last 5 billion years.
\end{abstract}

Key words: galaxies: starburst - infrared: galaxies - infrared: ISM

Online-only material: color figures

\section{INTRODUCTION}

Luminous $\left(10^{11}<L_{\operatorname{IR}(8-1000)}<10^{12} L_{\odot}\right)$ and ultra-luminous infrared galaxies $\left(L_{\mathrm{IR}(8-1000)}>10^{12} L_{\odot}\right.$, (U)LIRGs) are among the most important populations in studies of galaxy evolution. The origin of their extreme luminosities has been the focus of debate since their discovery by IRAS, however, it is now widely accepted that local $(z<0.26)$ ULIRGs are primarily powered by star formation (e.g., Genzel et al. 1998; Rigopoulou et al. 1999; Farrah et al. 2007). While ULIRGs in the local universe are rare (e.g., Lagache et al. 2005), they contribute significantly to the total IR energy density from redshifts 0.5 and above (e.g., Le Floc'h et al. 2005; Rodighiero et al. 2010). At redshifts $z>2$ submillimeter galaxies (SMGs) are considered to be the more luminous counterparts of local

\footnotetext{
* Herschel is an ESA space observatory with science instruments provided by European-led Principal Investigator consortia and with important participation from NASA.
}

ULIRGs (e.g., Blain et al. 2002). There are many indications, however, that local ULIRGs differ systematically from their high-redshift counterparts. Many authors (e.g., Papovich et al. 2007; Farrah et al. 2008; Muzzin et al. 2010; Swinbank et al. 2010) have found that high- $z$ ULIRGs have different spectral energy distributions, mid-infrared properties and extent of starforming regions when compared to local ones. More recently, Rujopakarn et al. (2011) compared physical scales of the starforming regions and concluded that high- $z$ ULIRGs are more akin to local star-forming galaxies rather than local ULIRGs.

The fine structure line [C $\mathrm{CI}]$ at $158 \mu \mathrm{m}$ is one of the brightest emission lines in the spectra of galaxies. [C II] traces gas exposed to far-ultraviolet (FUV) photons from OB stars with energies greater than $11.3 \mathrm{eV}$, the ionization potential of $\mathrm{C}^{0}$. In these photodissociation regions (PDRs), atomic molecular hydrogen and electrons can collisionally excite the ground state of $\mathrm{C}^{+}$ions producing [C II] which cools the gas. Early [C II] detections with the Kuiper Airborne Observatory in nearby galaxies showed that the line was bright, $0.1 \%-1 \%$ of the observed far-infrared 
(FIR) luminosity (e.g., Stacey et al. 1991; Crawford et al. 1985). Subsequent observations with the Infrared Space Observatory confirmed these findings but highlighted a deficit of the [C II] line in the highest luminosity systems such as local ULIRGs (e.g., Luhman et al. 1998, 2003).

Since most local ULIRGs are thought to be star formation dominated (e.g., Genzel et al. 1998; Rigopoulou et al. 1999) the $[\mathrm{C}$ II]-deficit appeared at odds with previous results and a number of explanations were put forward, including size of [C II] emitting regions, metallicity and dust content (e.g., Luhman et al. 1998, 2003). But recent detections of [C II] in luminous $z>1.5$ star-forming systems with Herschel (e.g., Ivison et al. 2010; Valtchanov et al. 2011; George et al. 2013) and other ground-based facilities (e.g., Hailey-Dunsheath et al. 2010; Stacey et al. 2010) revealed that their [C II]/FIR luminosity ratios are similar to local star-forming galaxies, much above the median values found for local ULIRGs. More recently, GraciaCarpio et al. (2011) found that the [C II]/ $L_{\mathrm{FIR}}$ ratio is inversely proportional to $L_{\mathrm{FIR}} / M\left(\mathrm{H}_{2}\right)$ for a fixed $L_{\mathrm{FIR}}$ for a sample of local starbursts and (U)LIRGs.

Here we present the first results of a survey of [C II] in a sample of $0.2<z<0.8$ (U)LIRGs. The redshift range $0.2<z<0.8$ represents a crucial phase in galaxy evolution: it is exactly in this range that the star formation density of the universe increases steeply, becoming essentially flat at $z>1.5$ (e.g., Magnelli et al. 2013; Bouwens et al. 2009). [C II] observations of galaxies in this cosmic epoch will establish the long-sought link between the local and high- $z$ universe and allow us to form a benchmark for future studies of [C II] at higher redshifts.

\section{SAMPLE SELECTION AND OBSERVATIONS}

\subsection{The Sample}

The primary goal of the survey is to investigate the properties of the interstellar medium (ISM) and in particular whether intermediate redshift (U)LIRGs are [C II]-deficient like their local counterparts. To construct the present sample we employed the Herschel Multi-tiered Extragalactic Survey (Oliver et al. 2012) photometric catalogs produced using a prior source extraction based on the position of known $24 \mu \mathrm{m}$ sources (Roseboom et al. 2010). We searched for sources that satisfied the following two criteria: (1) $S_{250}>150 \mathrm{mJy}$, a limit imposed to ensure detection of the source against the background emission of the telescope at $80 \mathrm{~K}$ and, (2) redshift in the range $0.2<$ $z<0.8$ so that we could observe at least one of the primary cooling lines [C II] $158 \mu \mathrm{m},[\mathrm{N}$ II] $205 \mu \mathrm{m}$ and [CI] $307 \mu \mathrm{m}$ in the $194-671 \mu \mathrm{m}$ range. These criteria resulted in the selection of 22 (U)LIRGs with $L_{\mathrm{IR}(8-1000)}>10^{11.5} L_{\odot}, 17$ of them with confirmed spectroscopic redshifts $\left(z_{\text {spec }}\right)$.

We have supplemented the FIR spectroscopic data with single-dish CO data using the IRAM $30 \mathrm{~m}$ and ESO APEX telescopes and, spatially resolved optical Integral field spectroscopic data using the Oxford-Swift integral field unit (Thatte et al. 2010) on Palomar. The present Letter focuses on 12 intermediate redshift (U)LIRGs with $z_{\text {spec }}$ for which the [C II] $158 \mu \mathrm{m}$ line falls in the Spectral and Photometric Imaging REceiver (SPIRE)-Fourier Transform Spectrometer (FTS) range. We note that the [O I] $63 \mu \mathrm{m}$ line does not fall in the FTS range, hence we assume that $[\mathrm{C} \mathrm{II}]$ is the primary cooling line in our sample. Measurements of the full sample and reports of the ancillary datasets and measurements will be presented in G. E. Magdis et al. (in preparation).

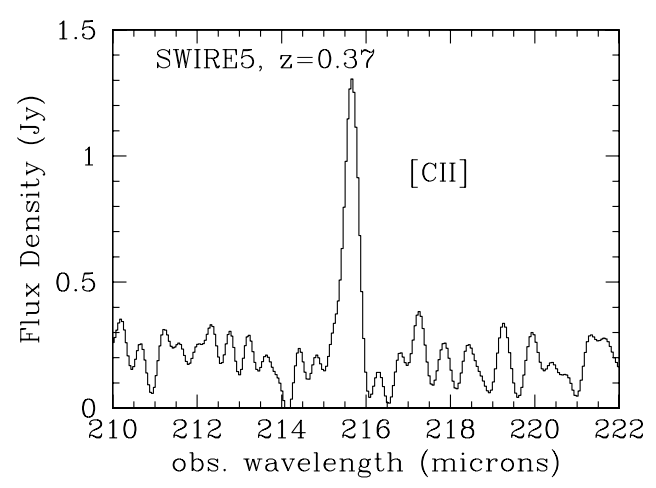

Figure 1. Continuum-subtracted region of the FTS spectrum around the [C II] line in the observed frame for one of our sample ULIRGs (SWIRE5) at $z=0.37$.

\subsection{SPIRE-FTS Spectroscopy}

The (U)LIRGs in our sample were observed with the SPIRE (Griffin et al. 2010) FTS on board the Herschel Space Observatory (Pilbratt et al. 2010), between 2012 March and 2013 January. The FTS observed 100 repetitions $(13,320 \mathrm{~s}$ total integration time) on each target in single pointing, high spectral resolution $\left(0.048 \mathrm{~cm}^{-1}\right)$ mode, with sparse spatial sampling. The SPIRE-FTS measures the Fourier transform of the spectrum of a source using two bolometer detector arrays, simultaneously covering wavelength bands of 194-313 $\mu \mathrm{m}$ (SSW) and 303-671 $\mu \mathrm{m}$ (SLW). All (U)LIRGs in the sample are pointlike, given the beam size in SSW and the distances. A typical spectrum around the $\left[\mathrm{C}_{\mathrm{II}}\right]$ line is shown in Figure 1. All ULIRGs in our sample are extremely faint targets for the FTS and, require post-pipeline processing beyond the standard reduction. A detailed account of the procedure used can be found in R. Hopwood et al. (in preparation). In brief, we used the standard FTS pipeline (T. Fulton et al., in preparation) in HIPE (Ott 2010) version 11 to reduce the data. Within the pipeline steps, a bespoke relative spectral response function constructed from selected long dark sky observations was applied if it improved the noise in the point source calibrated product (level 2 product).

Two methods can be used to remove residual background in the level 2 spectra. Firstly, spectra from detectors around the central "on-source" detector were selected and their average spectra subtracted from the central detector. The second method involves subtracting a dark sky spectrum observed on the same operational day. We found the off-axis subtraction provided the best reduction in residual for all but one observation. Once the optimum reduction method was determined and the level 2 spectra obtained, these were examined for spectral features. Since random noise in an FTS spectrum can easily mimic a faint line we used the jackknife technique as a reliability test to minimize spurious detections. In brief, each unaveraged level 2 spectrum, of 200 scans, was split into sequential subsets. This was repeated for subsets of decreasing number of scans. By plotting the averaged subsets at the expected line positions and making a visual comparison over all subsets, an assessment of the presence of an expected line was made.

Once a line was assessed as real, a bootstrap method was used to measure the line flux. For any given observation, scans were randomly sampled until the number in the parent population (of 200) was reached. These random scans were then averaged and the line measurements made by fitting a sinc function, or, if the line was partially resolved (in six cases) we employed a sinc convolved with a Gaussian. We repeated the process 
10,000 times for each observation and fitted a Gaussian to the resulting line flux distribution to obtain the mean line flux. The standard deviation was taken as the associated $1 \sigma$ uncertainties. Random frequency positions were also selected and the same process repeated to give comparison distributions and provide a second reliability check. Due to the nature of FTS random and systematic noise, the distributions obtained for random frequency positions are indistinguishable to those for faint lines $(<2 \sigma)$, however, a background level can be established with the bootstrapped results for these randomly selected positions, above which a real spectral feature is strongly suggested. For any line found to be below $2 \sigma$, the bootstrapped flux density is taken as an upper limit, but only if the presence of a [C II] line is supported by the jackknife and other visual checks.

\section{RESULTS}

\subsection{The $L_{\left[\mathrm{C}_{\mathrm{II}}\right.} / L_{\mathrm{IR}}$ Relationship}

We have detected [C II] line emission $(>3 \sigma)$ from 10 of the 12 ULIRGs with $z_{\text {spec }}$. The [C II] line is bright in all detected sources, in the range $(0.6-2.6) \times 10^{9} L_{\odot}$. The brightest source, with a [C II] line luminosity of $5.7 \times 10^{10} L_{\odot}$ has been identified with a $z=2.31$ luminous galaxy merger (XMM01; Fu et al. 2013) although the lens galaxy was originally selected. The two sources with the lowest [C II] line luminosity both contain spectroscopically confirmed active galactic nuclei (AGNs; Houck et al. 2005) but are not classified as QSOs.

The parameter $R=L_{[\mathrm{C} \text { II] }} / L_{\mathrm{FIR}}$, defined as the ratio of the [C II] line luminosity to the FIR continuum luminosity $L_{\mathrm{FIR}}{ }^{20}$ can be used to probe the strength of the ambient radiation field $\left(G_{0}\right.$, e.g., Kaufman et al. 1999) under the PDR paradigm. Since the emergent FIR intensity is directly proportional to the underlying radiation field and, $L_{\left[\mathrm{C}_{\mathrm{II}}\right]}$ is only weakly dependent on $G_{0}$ it follows that their ratio $R$ is inversely proportional to $G_{0}$. Low values of $R$ would imply a hard underlying radiation field. Despite local ULIRGs being predominantly starburst dominated (e.g., Rigopoulou et al. 1999; Farrah et al. 2007) their ratio $R=L_{\left[\mathrm{C}_{\mathrm{II}}\right]} / L_{\mathrm{FIR}}$ is surprisingly low, less than $1 \%$ (e.g., Luhman et al. 2003; Farrah et al. 2013). In contrast, high-redshift ULIRGs at $z \sim 1-2$ (e.g., Stacey et al. 2010) and $z>2$ lenses discovered by Herschel (e.g., Ivison et al. 2010; Valtchanov et al. 2011) have $R$ ratios similar to those of local star-forming galaxies.

In Figure 2 we plot the observed $R$ ratio as a function of $L_{\mathrm{FIR}}$, for a sample of nearby normal and starburst galaxies (Malhotra et al. 2001), local ULIRGs (Farrah et al. 2013; Diaz-Santos et al. 2013), high- $z$ star-forming and AGN-powered sources (Stacey et al. 2010; Hailey-Dunsheath et al. 2010), high- $z$ lenses from Ivison et al. (2010), Valtchanov et al. (2011), George et al. (2013), and our sample. Local star-forming and normal galaxies and high-redshift star-forming galaxies display ratios of $R \sim$ $0.001-0.01$ while $R$ is in the range $(1.3-10) \times 10^{-4}$ for local ULIRGs. The ratio varies between $R \approx 3 \times 10^{-4}-0.7 \times 10^{-2}$ for our sample (U)LIRGs. Turning to the two AGN sources in our sample with low $L_{\left[\mathrm{C}_{\text {II }}\right]} / L_{\mathrm{FIR}}$ we note that it is plausible that a sizeable fraction of the $L_{\mathrm{FIR}}$ originates in the AGN component hence lowering the overall $L_{[\mathrm{C} \text { II }]} / L_{\mathrm{FIR}}$ ratio.

Our finding reveals, for the first time, that intermediate redshift (U)LIRGs with no AGN have $L_{[\mathrm{C}}{ }_{\mathrm{II}} / L_{\mathrm{FIR}}$ ratios similar to those of high-redshift star-forming galaxies and local normal galaxies. The implications of this result for the nature and evolution of ULIRGs are discussed in Section 4.

\footnotetext{
${ }^{20}$ Defined as the luminosity between $42.5-122.5 \mu \mathrm{m}$.
}

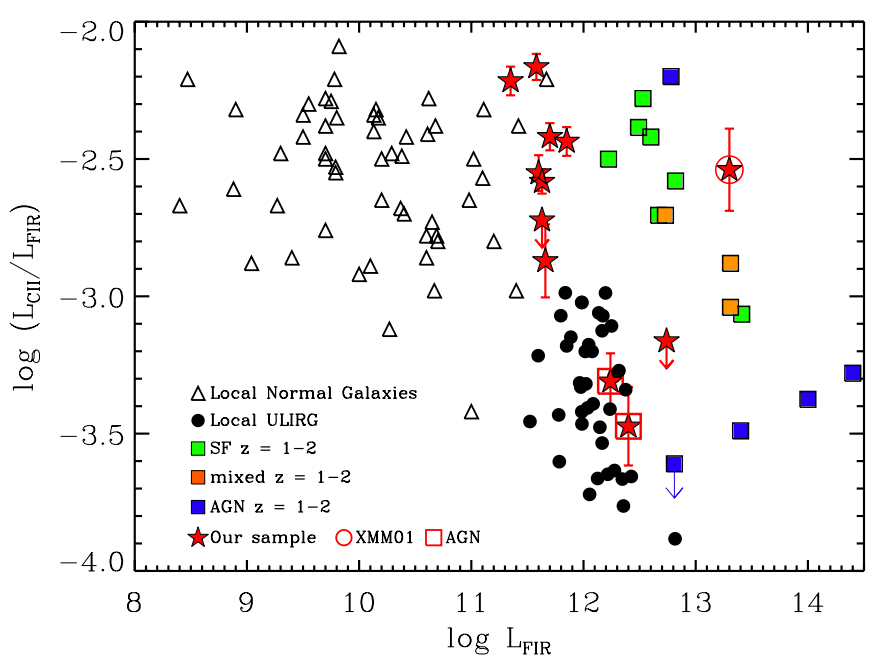

Figure 2. $\log \left(L_{[\mathrm{CII}]} / L_{\mathrm{FIR}}\right)$ as a function of $\log \left(L_{\mathrm{FIR}}\right)$ for local normal and starburst galaxies (open triangles) from Malhotra et al. (2001), local ULIRGs (black filled circles) from Farrah et al. (2013) and Diaz-Santos et al. (2013), highredshift $(1<z<2)$ star-forming galaxies (green squares), mixed systems (orange filled squares) and AGNs (blue filled squares) from Stacey et al. (2010). Red stars denote the 12 intermediate redshift (U)LIRGs presented in this work (circled star is $X M M 01$, squared stars denote AGNs). Note that $L_{\mathrm{FIR}(42.5-122.5 \mu \mathrm{m})}=$ $0.63 \times L_{\mathrm{IR}(8-1000 \mu \mathrm{m})}$.

(A color version of this figure is available in the online journal.)

\subsection{The $[C \mathrm{II}] / C O(1-0)$ Ratio and PDR Properties}

Early [C II] surveys of extragalactic sources and mapping of the Galaxy (e.g., Heiles 1994; Cubick et al. 2008) have established that a large fraction of [C II] emission in galaxies originates in PDRs on the outer layers of molecular clouds exposed to intense FUV radiation. [C II] also acts as a coolant of the low density warm ionized medium. Recent studies (e.g., Rigopoulou et al. 2013) found that up to $25 \%$ of the [C II] emission in the star-forming galaxy IC342 originates in the diffuse ionized gas. We will thus assume that the majority of [C II] emission in our sample (U)LIRGs originates in PDRs. Kaufman et al. (1999) presented PDR models in which the line emission from the clouds is determined by the density of the gas $\eta$ and the incident flux $G_{0}$ (expressed in units of the Habing field, $\left.1.6 \times 10^{-3} \mathrm{erg} \mathrm{cm}^{-2} \mathrm{~s}^{-1}\right)$. The models assume that the [C II] line and the FIR continuum are optically thin optically thick and self-absorption case is not likely (see, e.g., Luhman et al. 2003) but, the low-J CO transitions are optically thick. So when comparing models to observables one needs to subtract off the fraction of the [C II] line arising in the ionized medium ( 25\%, e.g., Rigopoulou et al. 2013), and multiply the CO line intensity by a factor of two for all objects.

Using the $\mathrm{CO}$ measurements reported in G. E. Magdis et al. (2013, in preparation) we examine the $\left.L_{[\mathrm{C}} \mathrm{II}\right] / L_{\mathrm{CO}(1-0)}$ ratio for our intermediate redshift ULIRGs. For sources without $\mathrm{CO}(1-0)$ measurements we use the conversion factors for SMGs $\mathrm{CO}(2-1) / \mathrm{CO}(1-0) \sim 0.84 \pm 0.13$ and $\mathrm{CO}(3-2) / \mathrm{CO}(1-0) \sim$ $0.52 \pm 0.09$ (e.g., Bothwell et al. 2013). The $L_{[\mathrm{C} \text { II }]} / L_{\mathrm{CO}(1-0)}$ ratio for our sample is $3300 \pm 420$. This value excludes the two sources that contain confirmed AGNs. For comparison, local ULIRGs have a mean $L_{[\mathrm{C} \text { II] }} / L_{\mathrm{CO}(1-0)}$ ratio of $1500 \pm 260$, high- $z$ star-forming galaxies $4050 \pm 410$, normal local galaxies $1800 \pm 270$, while starburst nuclei and local Galactic starforming regions have a ratio of $4100 \pm 320$ (Stacey et al. 1991). We find that the mean $L_{[\mathrm{C}}{ }_{\mathrm{II}} / L_{\mathrm{CO}(1-0)}$ value for our sample is a factor of two larger than that of local ULIRGs and closer 


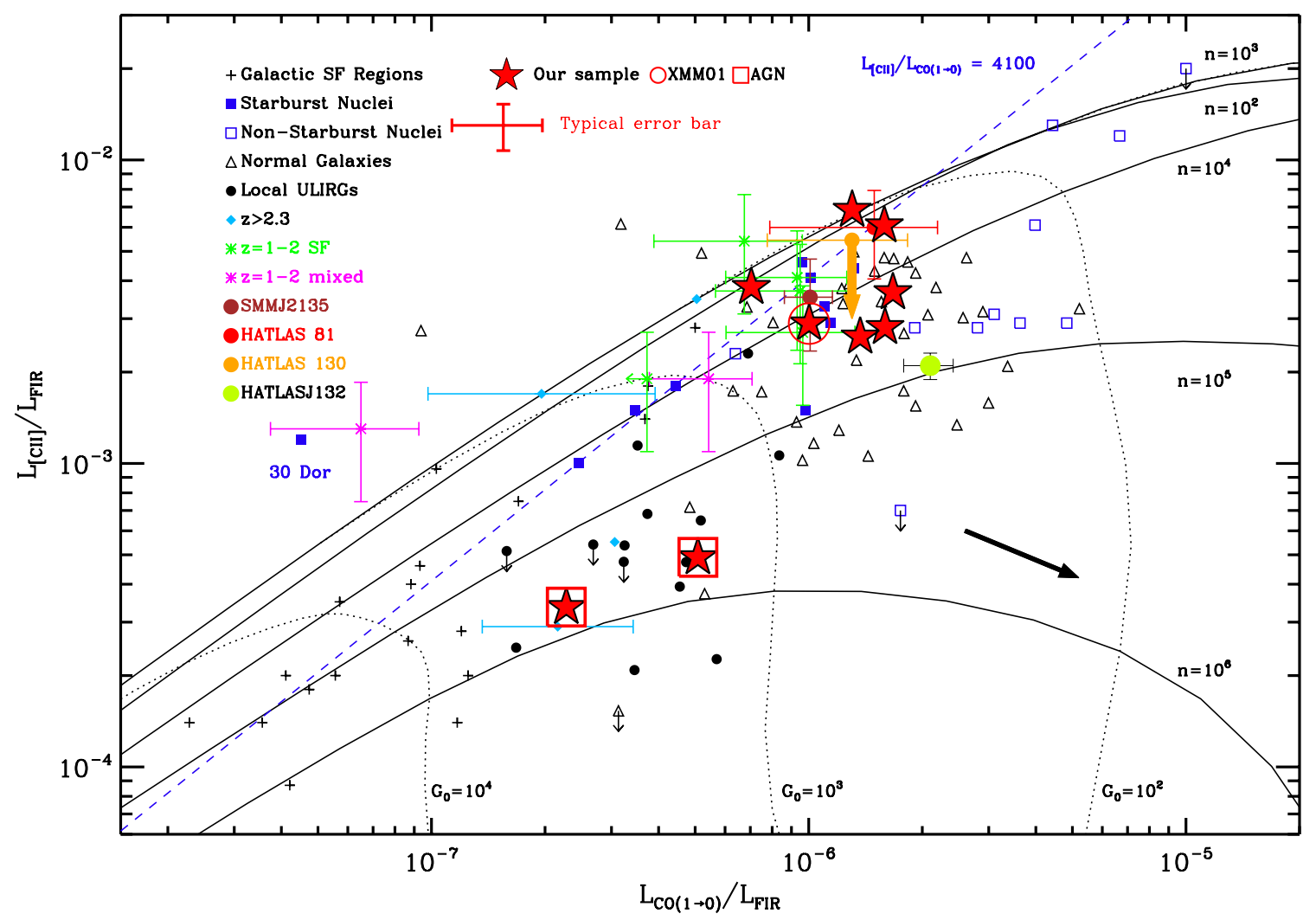

Figure 3. $L_{[\mathrm{C} \text { II] }} / L_{\mathrm{FIR}}$ as a function of $L_{\mathrm{CO}(1-0)} / L_{\mathrm{FIR}}$ for Galactic star-forming regions (crosses), local starburst nuclei (filled squares), local non-starburst nuclei (open squares), local normal galaxies (triangles), local ULIRGs (circles), redshift 1-2 sources (asterisks with error bars), high- $z$ sources (cyan diamonds), adapted from Hailey-Dunsheath et al. (2010). Herschel lenses are from: SMMJ2135 (Ivison et al. 2010), HATLAS 81 and HATLAS 130 (Valtchanov et al. 2011 ), HATLASJ 132 (George et al. 2013). Red asterisks denote the present sample (circled star is XMM01, squared stars denote AGNs). The typical error bar shown refers to our sample only. Overplotted are the PDR model values for $\eta$ and $G_{0}$ from Kaufman et al. (1999). The plot is based on observed values and is intended as a first order diagnostic tool, hence does not include any correction. The black arrow indicates the direction that all data points in the plot will shift when corrections are applied (see text).

(A color version of this figure is available in the online journal.)

to the value found for local and high- $z$ star-forming galaxies. Although local normal galaxies and local ULIRGs have similar $L_{[\mathrm{C} \text { II] }} / L_{\mathrm{CO}(1-0)}$ ratios, their $L_{[\mathrm{C}}{ }_{\text {II] }} / L_{\mathrm{FIR}}$ ratios are a factor of 10 different. Local normal galaxies have higher $L_{[\mathrm{C} \text { II] }} / L_{\mathrm{FIR}}$ values than those seen in local ULIRGs. Likewise, the $L_{[\mathrm{C} \text { II] }} / L_{\mathrm{CO}(1-0)}$ and $L_{[\mathrm{C} \text { II] }} / L_{\mathrm{FIR}}$ ratios of local and intermediate redshift ULIRGs are different by a factor of 2 and 10 , reinforcing our earlier findings that the properties of the ISM of ULIRGs changes dramatically between $z=0$ and $z=0.5$.

To further investigate these trends, in Figure 3 we plot the $L_{[\mathrm{CI}} / L_{\mathrm{FIR}}$ and $L_{\mathrm{CO}(1-0)} / L_{\mathrm{FIR}}$ ratios for our sample and compare them to those of galactic star-forming regions, local ULIRGs, local normal galaxies, high- and low- $z$ star-forming galaxies and recent Herschel measurements of high- $z$ lensed star-forming galaxies from Ivison et al. (2010), Valtchanov et al. (2011), and George et al. (2013). For reference, we also show PDR model calculations ${ }^{21}$ for gas density $\eta$ and FUV strength $G_{0}$ (adapted from Hailey-Dunsheath et al. 2010) applicable to sources without a dominant AGN component.

The mean $L_{\text {[C II] }} / L_{\mathrm{FIR}}$ and $L_{\mathrm{CO}(1-0)} / L_{\mathrm{FIR}}$ ratios for intermediate redshift ULIRGs are a factor of 10 and seven times higher than those of local ULIRGs. Since the emergent $L_{\mathrm{FIR}}$ is proportional to the incident radiation flux (parameterized by $G_{0}$ ) the smaller $L_{[\mathrm{C} \text { II] }} / L_{\mathrm{FIR}}$ line ratios observed in local ULIRGs indicate that [C $\mathrm{CI}]$ must be produced in dense PDRs illuminated by a strong radiation field (e.g., Farrah et al. 2013). In a recent

$\overline{21}$ Based on the PDRToolbox Web site: http://dustem.astro.umd.edu/. study of [C II] line emission from a sample of local ULIRGs Diaz-Santos et al. (2013) argue in favor of smaller/compact star-forming regions.

Clearly, none of the above scenarios are applicable to the intermediate redshift (U)LIRGs. The data points for our sample fall in the range $10^{3}<G_{0}<10^{2}$ an area which overlaps with the low end of the distribution of the $G_{0}$ values for starbursts and the high end of $G_{0}$ values for normal galaxies. Hence, we conclude that the bulk of the molecular gas in intermediate redshift (U)LIRGs is exposed to a softer radiation field than that of local ULIRGs, more akin to those found in nearby starburst nuclei.

The $L_{\mathrm{CO}(1-0)} / L_{\mathrm{FIR}}$ of intermediate redshift (U)LIRGs is $\sim 7$ times higher than that of local ULIRGs. It is similar to the upper end of the values seen in local starburst nuclei and local normal galaxies. However, as we discussed earlier, intermediate redshift ULIRGs display an $L_{[\mathrm{C} \text { II] }} / L_{\mathrm{CO}(1-0)}$ ratio of 3000 which is higher than the value found in local normal galaxies but lower that that of starburst nuclei (4100). A lower $L_{[\mathrm{C} \text { II] }} / L_{\mathrm{CO}(1-0)}$ (for the same $L_{\mathrm{CO}(1-0)} / L_{\mathrm{FIR}}$ value) ratio would imply that most of the $\mathrm{CO}$ emission originates in molecular clouds residing in less active star-forming regions than those responsible for producing the fine-structure emission lines.

\section{THE NATURE AND EVOLUTION OF LUMINOUS INFRARED GALAXIES}

The star formation rate density (SFRD) in the universe increases dramatically from the present day to $z \sim 1$ at 
which point it becomes flat out to $z \sim 3-4$ (e.g., Bouwens et al. 2009; Magnelli et al. 2013). LIRGs are a dominant component to the co-moving SFRD between $z \sim 0.5-1$ so their properties may give clues to the drivers behind the dramatic rise at $z<1$.

Although local ULIRGs have been used as templates for $z \sim$ 2 LIRGs, increasing evidence exists (e.g., Papovich et al. 2007; Farrah et al. 2008; Swinbank et al. 2010) that they are distinctly different to their high- $z$ counterparts.

It is now well established that the intense star-forming activity of local ULIRGs is the result of merging (e.g., Sanders \& Mirabel 1996). Morphology indications in high-resolution Hubble Space Telescope images however, reveal that, at $z \sim 2$ at least $50 \%$ of the Herschel-selected starbursts are not driven by galaxy mergers (e.g., Kartaltepe et al. 2012). In Section 3 we presented new evidence that the properties of the starforming regions of our intermediate redshift (U)LIRGs are distinctly different to those of local ULIRGs. Instead, they bear a closer resemblance to those found in high-redshift star-forming galaxies. In particular, we found evidence that the star-forming regions of our $z \sim 0.5(\mathrm{U}) \mathrm{LIRG}$ are illuminated by moderately intense FUV radiation, with $G_{0}$ in the range $10^{2}-10^{2.5}$ and $G_{0} / \eta \sim 0.1-1 \mathrm{~cm}^{3}$ (when the appropriate corrections of $0.7 \times L_{[\mathrm{C} \text { II }]}$ and $2 \times L_{\mathrm{CO}}$ are applied). Similar $G_{0} / \eta$ values have been found for local normal and starburst galaxies (e.g., Wolfire et al. 1990). In contrast, the low $L_{\left[\mathrm{C}_{\text {II }}\right]} / L_{\mathrm{FIR}}$ values seen in local ULIRGs are the result of the presence of intense radiation fields $G_{0}$ with high column densities $G_{0} / \eta \sim 0.02-0.03 \mathrm{~cm}^{3}$ (Farrah et al. 2013) and possibly compact sizes (e.g., Diaz-Santos et al. 2013). The high $L_{\left[\mathrm{C}_{\text {II }}\right.} / L_{\mathrm{FIR}}$ values seen in intermediate redshift ULIRGs together with the modest radiation fields speak against the existence of such dense compact PDRs, hence the starforming regions of intermediate redshift (U)LIRGs must be extended.

Our survey has revealed a strong evolution in the properties of ULIRGs at $0.2<z<0.8$ and confirms the use of the [C II] line and the $L_{\left[\mathrm{C}_{\mathrm{II}}\right]} / L_{\mathrm{IR}}$ and $L_{\left[\mathrm{CC}_{\mathrm{II}}\right]} / L_{\mathrm{CO}(1-0)}$ ratios, in probing the properties of the star-forming regions in galaxies at low, intermediate and high redshifts. Even at modest redshifts $(z \sim$ $0.5)$ the nature of the ULIRG population changes significantly from exclusively compact merger-driven to a more varied population.

We thank the anonymous referee for his/her insightful comments. D.R. and G.E.M. acknowledge support from grant ST/K00106X/1 and the John Fell Oxford University Press (OUP) Research Fund (G.E.M.). This Letter is based on data from Herschel's SPIRE-FTS. SPIRE has been developed by a consortium of institutes led by Cardiff University (UK) and including: University of Lethbridge (Canada); NAOC (China); CEA, LAM (France); IFSI, University of Padua (Italy); IAC (Spain); Stockholm Observatory (Sweden); Imperial College
London, RAL, UCL-MSSL, UKATC, University of Sussex (UK); and Caltech, JPL, NHSC, University of Colorado (USA). This development has been supported by national funding agencies: CSA (Canada); NAOC (China); CEA, CNES, CNRS (France); ASI (Italy); MCINN (Spain); SNSB (Sweden); STFC, UKSA (UK); and NASA (USA).

Facilities: Herschel, IRAM:30m, APEX

\section{REFERENCES}

Blain, A. W., Smail, I., Ivison, R. J., Kneib, J-P., \& Frayer, D. T. 2002, PhR, 369,111

Bothwell, M. S., Smail, I., Chapman, S. C., et al. 2013, MNRAS, 429, 3047

Bouwens, R. J., Illingworth, G. D., Franx, M., et al. 2009, ApJ, 705, 936

Crawford, M. K., Genzel, R., Townes, C. H., \& Watson, D. M. 1985, ApJ, 291, 755

Cubick, M., Stutzki, J., Ossenkopf, V., Kramer, C., \& Rollig, M. 2008, A\&A, 488, 623

Diaz-Santos, T., Armus, L., Charmandaris, V., et al. 2013, ApJ, 774, 68

Farrah, D., Bernard-Salas, J., Spoon, H. W. W., et al. 2007, ApJ, 667, 149

Farrah, D., Lebouteiller, V., Spoon, H., et al. 2013, ApJ, 773, 38

Farrah, D., Lonsdale, C. J., Weedman, D. W., et al. 2008, ApJ, 677, 957

Fu, H., Cooray, A., Feruglio, C., et al. 2013, Natur, 498, 338

Genzel, R., Lutz, D., Sturm, E., et al. 1998, ApJ, 498, 579

George, R. D., Ivison, R. J., Hopwood, R., et al. 2013, MNRAS, 436, L99

Gracia-Carpio, J., Sturm, E., Hailey-Dunsheath, S., et al. 2011, ApJ, 728, 7

Griffin, M. J., Abergel, A., Abreu, A., et al. 2010, A\&A, 418, L3

Hailey-Dunsheath, S., Nikola, T., \& Stacey, G. J. 2010, ApJ, 714, 162

Heiles, C. 1994, ApJ, 436, 702

Houck, J. R., Soifer, B. T., Weedman, D., et al. 2005, ApJ, 622, 105

Ivison, R. J., Swinbank, A. M., Swinyard, B.M, et al. 2010, A\&A, 518, 35

Kartaltepe, J. S., Dickinson, M., Alexander, D., et al. 2012, ApJ, 757, 23

Kaufman, M. J., Wolfire, M. G., Hollenbach, D. J., \& Luhman, M. 1999, ApJ, 527,795

Lagache, G., Puget, J.-L., \& Dole, H. 2005, ARA\&A, 43, 727

Le Floc'h, E., Papovich, C., Dole, H., et al. 2005, ApJ, 632, 169

Luhman, M. L., Satyapal, S., Fischer, J., et al. 1998, ApJL, 504, L11

Luhman, M. L., Satyapal, S., Fischer, J., et al. 2003, ApJL, 594, L758

Magnelli, B., Poppeso, P., Berta, S., et al. 2013, A\&A, 553, 112

Malhotra, S., Kaufman, M. J., Hollenbach, D., et al. 2001, ApJ, 561, 766

Muzzin, A., van Dokkum, P., Kriek, M., et al. 2010, ApJ, 725, 742

Oliver, S. J., Bock, J., Altieri, B., et al. 2012, MNRAS, 424, 1614

Ott, S. 2010, in ASP Conf. Ser. 434, Astronomical Data Analysis Software and Systems XIX, ed. Y. Mizumoto, K.-I. Morita, \& M. Ohishi (San Francisco, CA: ASP), 139

Papovich, C., Rudnick, G., Le Floc'h, E., et al. 2007, ApJ, 668, 45 Pilbratt, G. L., Riedinger, J. R., Passvogel, T., et al. 2010, A\&A, 518, 1 Rigopoulou, D., Hurley, P., Swinyard, B. M., et al. 2013, MNRAS, 434, 2015 Rigopoulou, D., Spoon, H. W. W., Genzel, R., et al. 1999, AJ, 118, 2625

Rodighiero, G., Cimatti, A., Gruppioni, C., et al. 2010, A\&A, 518, 25

Roseboom, I. G., Oliver, S. J., Kunz, M., et al. 2010, MNRAS, 409, 48

Rujopakarn, W., Rieke, G. H., Eisenstein, D. J., \& Juneau, S. 2011, ApJ, 726, 93

Sanders, D. B., \& Mirabel, I. F. 1996, ARA\&A, 34, 749

Stacey, G. J., Geis, N., Genzel, R., et al. 1991, ApJ, 373, 423

Stacey, G. J., Hailey-Dunsheath, S., Ferkinhoff, C., et al. 2010, ApJ, 724,957

Swinbank, A. M., Smail, I., Chapman, S. C., et al. 2010, MNRAS, 405, 234

Thatte, N., Tecza, M., Clarke, F., et al. 2010, Proc. SPIE, 7735, 14

Valtchanov, I., Virdee, J., Ivison, R. J., et al. 2011, MNRAS, 415, 3473

Wolfire, M. G., Tielens, A. G. G. M., \& Hollenbach, D. 1990, ApJ, 358, 116 